Reprod. Nutr. Dévelop., 1983, 23 (5), 837-846.

\title{
Influence d'un mode d'élevage sur la mise en réserve des graisses par le tissu adipeux chèz les ovins
}

\author{
A. VÉZINHET, J. NOUGUES, J. TEYSSIER
}

avec la collaboration technique de E. BOUTHIER, Thérèse CHERY, Odette FRECCERO, G. REBOUL

Station de Physiologie animale, I.N.R.A.,

E.N.S.A., Place Viala, 34060 Montpellier Cedex, France.

Summary. Effect of the rearing method on lipid deposition in the adipose tissue of sheep.

Adipocyte synthesis de novo and lipoprotein lipase acitivity have been used simultaneously to measure the lipogenic activity of adipose tissue in sheep. Acetate and glucose were used as precursors of fatty acid synthesis. The sheep were raised either outdoors or in a sheepfold. They were slaughtered by lots at mean weights of 24 and $32.5 \mathrm{~kg}$.

Compared to lipoprotein lipase activity, de novo synthesis of fatty acids was the main way of constituting lipid depositions. Raising the sheep outdoors favored the use of glucose as precursor of lipid synthesis at the first slaughter stage at $24 \mathrm{~kg}$. Later at $32.5 \mathrm{~kg}$, glucose utilization was practically zero compared to acetate, whatever the mode of rearing. The NADPH production needed for fatty acid synthesis was almost entirely due to NADP isocitrate dehydrogenase activity. Variations in both de novo synthesis and in lipoprotein lipase activity in relation with rearing method and slaughter weight were especially evident in the group raised outdoors.

\section{Introduction.}

La participation du tissu adipeux à la mise en réserve des graisses a suscité depuis plusieurs années un très vif intérêt. La cellule adipeuse s'est révélée capable d'assurer la synthèse des lipides. On a d'abord montré qu'elle pouvait utiliser le glucose comme source de carbone (Martin et Vagelos, 1965, Flatt, 1970, Ballard, Filsell et Jarret, 1972). D'autres précurseurs comme l'acétate, le propionate et le lactate peuvent jouer ce rôle notamment chez le ruminant (Hanson et Ballard, 1967, Young, Thorp et de Lumen, 1969, Ingle, Bauman et Garrigus, 1972 a et $b$, Pothoven et Beitz, 1973). Les enzymes clefs de la lipogenèse ont été progressivement étudiées. On a aussi montré que l'âge des animaux, leur état nutritionnel, le type de dépôt, le nombre et la taille des adipocytes, pourraient influencer la lipogenèse. Mais la synthèse de novo n'est pas seule à permettre la 
mise en réserve des graisses. L'enzyme lipoprotéine lipase (LPL) assure la captation par l'adipocyte des triglycérides circulants après leur hydrolyse. La LPL est abondante dans le tissu adipeux où elle est synthétisée, avant de se fixer à l'endothélium des capillaires voisins. Sa mise en évidence est due, entre autres, aux travaux de Bezman, Felts et Havel (1962), Robinson (1963), Garfinkel, Baker et Schotz (1967), Scow et al. (1972). Ainsi la mesure de l'activité de la LPL est essentielle pour apprécier la capacité de mise en réserve des graisses par le tissu adipeux.

Paradoxalement, peu de travaux ont été consacrés à l'étude simultanée de la synthèse de novo des lipides par l'adipocyte et de l'activité LPL. C'est ce type d'étude que nous avons réalisé chez des ovins en croissance élevés en plein air et en bergerie.

\section{Matériel et méthodes.}

Notre étude a été effectuée sur 16 agneaux mâles Mérinos d'Arles, dont 7 étaient élevés en plein air permanent sur des parcelles de garrigues avec une charge moyenne de 4 animaux par hectare. Les 9 autres étaient en stabulation permanente. Les agneaux ont été abattus par paires - un agneau plein air et un en bergerie - le même jour à la même heure. Deux poids moyens d'abattage ont été retenus : 24 et $32,5 \mathrm{~kg}$, avec 8 agneaux sacrifiés à chacun de ces stades. Compte tenu de la variabilité individuelle des poids vifs, l'âge des agneaux abattus à $24 \mathrm{~kg}$ était compris entre 70 et 90 jours. Pour ceux abattus à $32,5 \mathrm{~kg}$ il variait de 100 à 120 jours.

Alimentation. - Les animaux élevés en plein air sont restés, quel que soit le lot, en présence de leur mère; ils ont donc reçu une alimentation lactée et se sont progressivement adaptés à la pâture de la végétation naturelle des garrigues.

Les animaux élevés en bergerie sont également restés avec leurs mères et recevaient, outre le lait, un foin de luzerne d'excellente qualité ainsi qu'un concentré du commerce dosant $12 \%$ de matières protéiques brutes dans la matière sèche. Jusqu'au moment du sacrifice les animaux avaient un accès libre à la nourriture.

Prélèvements des tissus adipeux. - Ils ont été effectués à heure fixe le matin, entre 8 h 30 et 9 h 30, tout de suite après le sacrifice. Un échantillon de tissu sous-cutané était prélevé dans la région de l'aine, un autre provenait du tissu adipeux interne de la région du grand épiploon (omentum majus). Les mesures directes de la synthèse de novo des lipides et de la LPL étaient effectuées immédiatement. La mesure des activités enzymatiques liées à la synthèse de novo était réalisée sur des échantillons conservés à $-80^{\circ} \mathrm{C}$.

Mesure de la synthèse de novo des lipides. - Elle a été réalisée sur adipocytes isolés selon la méthode de Rodbell (1964). Le milieu d'incubation et les techniques analytiques d'extraction et de dosage des lipides et des acides gras ont 
été précédemment décrits (Vézinhet, 1976). La synthèse de novo des lipides a été mesurée à partir de l'incorporation de deux précurseurs, glucose et acétate, avec l'emploi d'éléments marqueurs : glucose $U^{14} \mathrm{C}$ et acétate $1^{14} \mathrm{C}$.

Concernant les dosages d'enzymes liés à la synthèse de novo, l'activité de l'acetyl-CoA-carboxylase a été mesurée par la méthode de Chang et al. (1965) modifiée par Chakrabarty et Leveille (1969). La production de NADPH nécessaire à la biosynthèse des acides gras a été appréciée par la mesure des activités de l'enzyme malique selon Hsu et Lardy (1969) et de l'enzyme isocitrate deshydrogenase (ICDH) d'après la méthode de Bergmeyer (1963).

Méthode d'extraction et mesure de l'activité de la LPL. - La technique adoptée est celle des homogénats aqueux utilisée par Chilliard, Dorléans et Fehr (1977) légèrement modifiée. L'homogénéisation est réalisée dans un tampon ammoniacal hépariné. Environ $800 \mathrm{mg}$ de tissu adipeux sont broyés au broyeur Ultraturax ( 2 fois 10 à $50000 \mathrm{tr} / \mathrm{min}$ ). Le broyat est repris dans $4 \mathrm{ml}$ du même tampon. Une légère centrifugation permet d'éliminer les matières grasses et les éléments de la trame conjonctivo-vasculaire. L'extrait aqueux est recueilli et mis en incubation en présence d'une émulsion de lipides à $10 \%$. L'activité de la LPL est mesurée par la libération des acides gras libres dosés selon Dole et Meinertz (1960) elle est exprimée en nmoles libérées par $\mathrm{g}$ de tissu et par minute. Dans le but d'étudier l'activité enzymatique par le nombre de cellules ( $10^{6}$ adipocytes) l'activité par $\mathrm{g}$ de tissu a été divisée par le nombre d'adipocytes contenus dans $1 \mathrm{~g}$ de tissu adipeux. La mesure du nombre des adipocytes a été effectuée selon une méthode précédemment décrite (Nougues et Vézinhet, 1977).

Analyse des résultats. - Les données recueillies sur le tissu adipeux se caractérisent par une forte variabilité. En outre, les échantillons étudiés sont de faible taille. La discussion sur les moyennes arithmétiques est réduite à une simple description des relations d'ordre (partiel) qu'elles font apparaître entre échantillons. Nous avons refusé, à ce niveau, de prendre une décision sur la base de deux échantillons car le test $t$ de Student est inapplicable.

Pour décider si les populations dont les échantillons sont extraits diffèrent entre elles, nous avons retenu une analyse de rang ou analyse non paramétrique. II s'agit du test de Mann-Whitney (test bilatéral) qui autorise une discussion des résultats indépendamment des paramètres calculés par ailleurs, comme la moyenne arithmétique.

\section{Résultats.}

Synthèse de novo des lipides. - Les figures 1 et 2 montrent que les adipocytes des deux dépôts étudiés sont capables de convertir l'acétate et le glucose en lipides et en acides gras. Ils le font cependant à des intensités très variables, l'acétate étant un bien meilleur précurseur de la synthèse des acides gras que le glucose. En outre, la totalité de l'acétate incorporé dans les lipides se retrouve dans les acides gras.

La situation est différente pour le glucose dont une importante proportion est incorporée dans les lipides sous forme de glycérol comme en témoignent les 
écarts de moyennes enregistrés entre l'incorporation dans les lipides et les acides gras. L'influence du type de dépôt utilisé est faible quel que soit le mode d'élevage. On note cependant une activité de synthèse supérieure, pour les agneaux du premier stade, dans le tissu Omental comparativement au tissu sous-cutané (fig. 1). Au deuxième stade, c'est au contraire le tissu sous-cutané qui manifeste l'activité la plus forte (fig. 2).

Influence du mode d'élevage. - Elle est très importante dans l'étude réalisée au premier stade (fig. 1). Qu'il s'agisse de l'utilisation du glucose ou de l'acétate comme précurseur de la synthèse des acides gras, les agneaux élevés en plein air ont toujours manifesté une activité lipogénique bien supérieure à celle des agneaux de bergerie pour les deux dépôts utilisés (omental et sous-cutané). Le test de Mann-Whitney a toujours mis en évidence des différences significatives (fig. 1).

Les résultats obtenus au cours du premier stade font aussi apparaître que le glucose est mieux utilisé, comme précurseur de la synthèse des acides gras, en élevage de plein air comparé à celui en bergerie. On constate (fig. 1) que pour les agneaux de plein air, 20 à $25 \%$ du glucose incorporé dans les lipides se
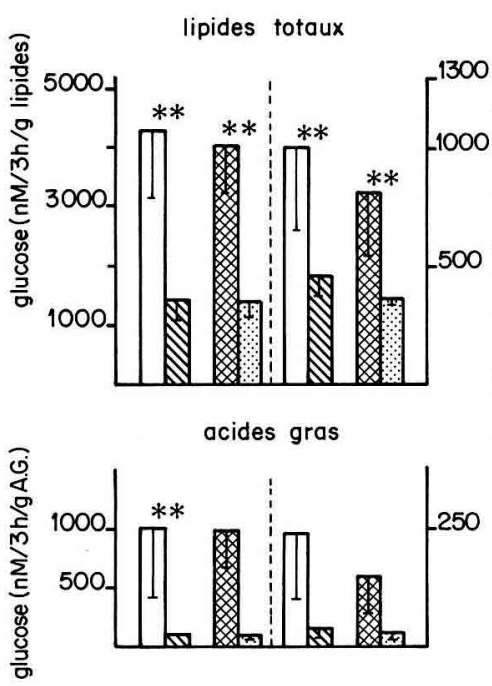

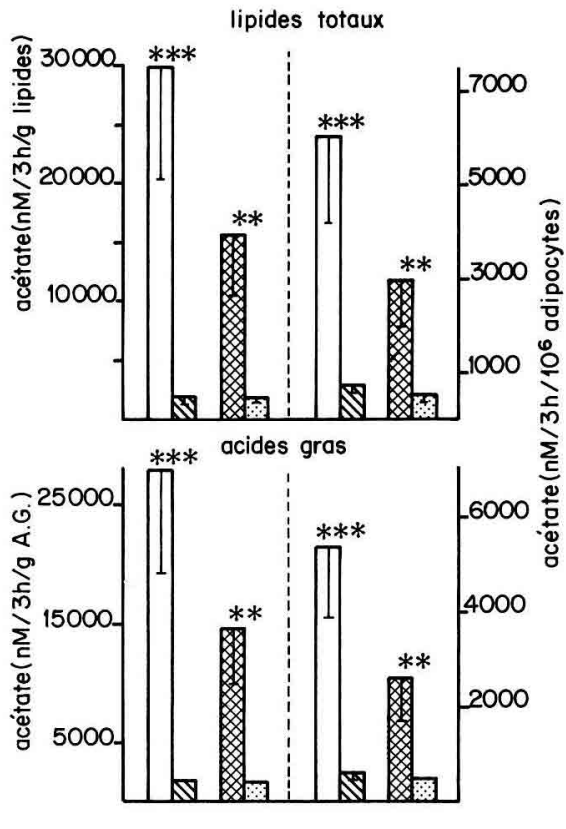

FIG. 1. - Synthèse de novo des lipides et acides gras: conversion de glucose et d'acétate dans les dépôts adipeux d'agneaux élevés en plein air ou en bergerie et sacrifiés à $24 \mathrm{~kg}$. L'incorportation mesurée (moyenne \pm erreur-type) est rapportée soit au gramme de lipides ou d'acides gras, soit à $10^{6}$ adipocytes isolés.

Agneaux de plein air : $\square$, tissu omental ; tissu sous-cutané.

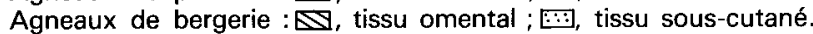

La comparaison par le test de Mann-Whitney des différences observées entre les modes d'élevage est représentée par les valeurs de $\alpha$ aux seuils suivants: ${ }^{* *} 0,02<\alpha<0,05$; ${ }^{*} 0,05<\alpha<0,10$; NS $\alpha<0,10$. 
retrouve dans les acides gras. Le pourcentage est de l'ordre de 5 à $10 \%$ pour ceux élevés en bergerie.

Au deuxième stade d'étude (fig. 2), l'utilisation du glucose devient très faible chez les agneaux de plein air ( 3 à $5 \%$ ). Elle est par contre plus élevée chez les agneaux de bergerie (15 à $20 \%$ ).

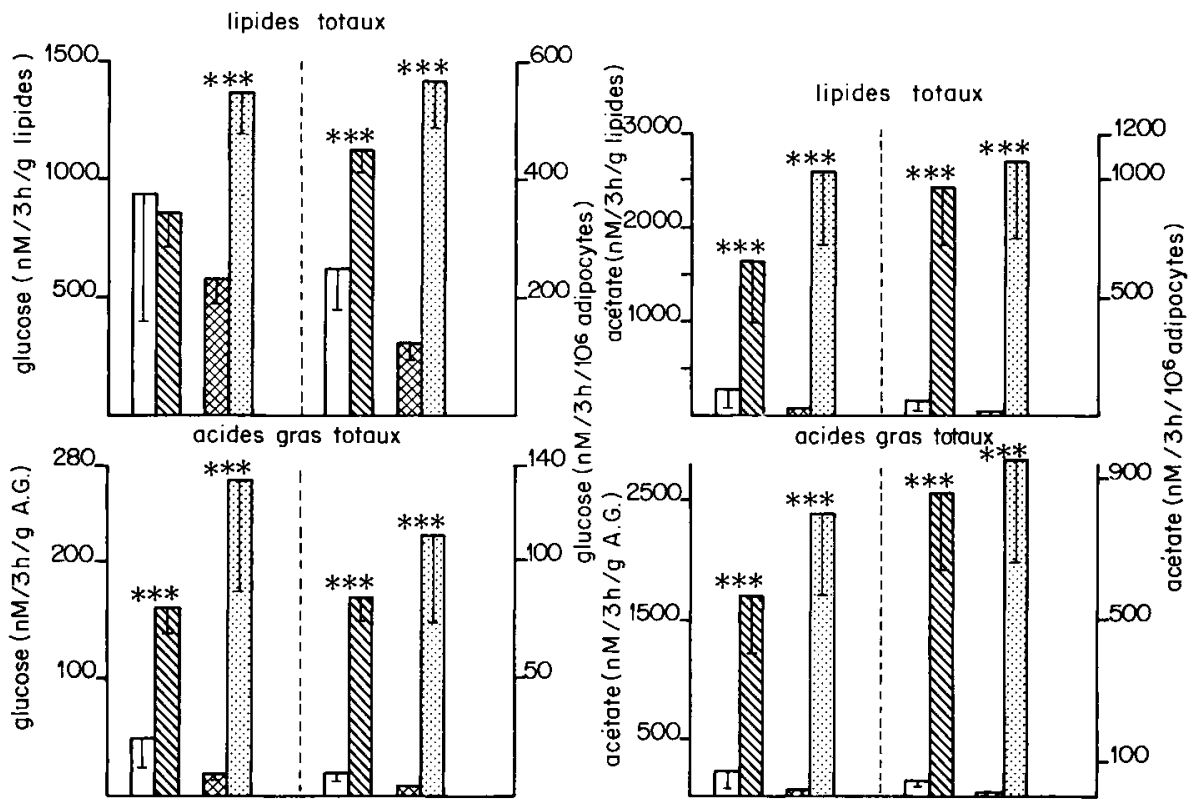

FIG. 2. - Synthèse de novo des lipides et acides gras: conversion de glucose et d'acétate dans les dépôts adipeux d'agneaux élevés en plein air ou en bergerie et sacrifiés à $32,5 \mathrm{~kg}$. L'incorporation mesurée (moyenne \pm erreur-type) est rapportée soit au gramme de lipides ou d'acides gras, soit à $10^{6}$ adipocytes isolés.

Légende et

Comparaison par le test de Mann-Whitney : voir figure 1.

Toujours au deuxième stade, on constate une lipogenèse bien supérieure chez les animaux de bergerie, ce qui est l'inverse de la situation du premier stade. II convient cependant de noter que cela est essentiellement le fait d'une forte réduction de la lipogenèse chez les agneaux de plein air, tandis que chez les agneaux de bergerie elle se maintient à un niveau sensiblement du même ordre qu'au premier stade. Ainsi l'utilisation de l'acétate au deuxième stade ne représente plus que 0,5 à $1 \%$ de celle enregistrée au premier stade dans le cas des agneaux de plein air.

La comparaison des activités enzymatiques liées à la lipogenèse (fig. 3) met en évidence, au premier stade d'étude, une activité de l'acétyl-CoA-carboxylase faible et du même ordre de grandeur. Quel que soit le mode d'élevage et le type de dépôt, il n'y a jamais de différence significative (test de Mann-Whitney). Par contre, au deuxième stade, les agneaux élevés en bergerie ont une activité 
actétyl-CoA-carboxylase toujours significativement plus forte que ceux élevés en plein air.

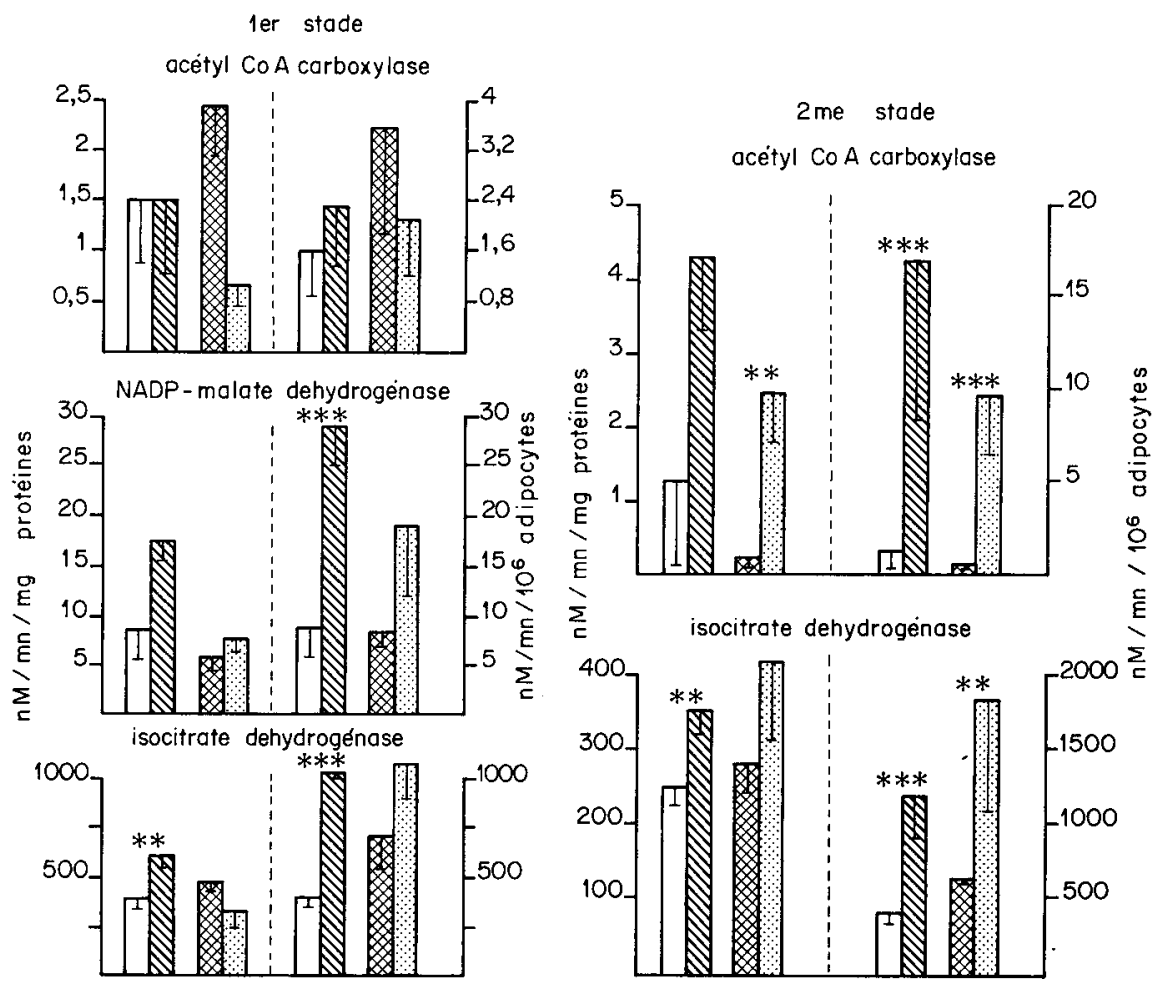

FIG. 3. - Synthèse de novo des lipides et acides gras : comparaison des activités enzymatiques (Acétyl-CoA carboxylase, NADP Malate déshydrogénase et NADP Isocitrate déshydrogénase) chez des agneaux élevés en plein air ou en bergerie et sacrifiés à $24 \mathrm{~kg}$ (premier stade) ou $32,5 \mathrm{~kg}$ (deuxième stade). Les activités sont rapportées par minute (moyenne \pm erreur-type) soit au $\mathrm{mg}$ de protéines soit à $10^{6}$ adipocytes.

Légende et

Comparaison par le test de Mann-Whitney : voir figure 1.

En ce qui concerne l'activité de production de NADPH, estimée par la NADP malate déshydrogénase (EM) et l'isocitrate déshydrogénase (ICDH), l'essentiel de cette production est liée à l'activité de l'ICDH. L'enzyme malique (EM) faible au premier stade étudié, n'a même pas pu être dosée au deuxième stade. Pour I'ICDH, dans tous les cas, ce sont les animaux élevés en bergerie qui ont manifesté l'activité la plus importante. Dans le cas du tissu omental, les différences enregistrées sont toujours significativement plus fortes pour les agneaux de bergerie. Dans le cas du tissu sous-cutané, les différences, sans être toujours significatives, témoignent d'une activité plus forte chez les agneaux de bergerie.

Activité de la lipoprotéine lipase. - L'étude relative à l'activité de la LPL a tout d'abord montré que cette activité est importante et vient s'ajouter à l'activité de synthèse de novo pour assurer la mise en réserve des graisses. 
La figure 4 montre qu'au premier stade l'activité LPL des agneaux élevés en plein air est, comme l'était la synthèse de novo, toujours significativement plus forte quand elle est exprimée par rapport à $1 \mathrm{~g}$ de tissu adipeux et ceci quel que soit le dépôt. Les activités LPL sont par contre très voisines quand elles sont exprimées par rapport à $10^{6}$ adipocytes, ce qui souligne l'importance du paramètre choisi pour l'interprétation des résultats.

Au deuxième stade d'étude, les animaux de bergerie montrent les activités LPL les plus fortes. Les différences observées sont dans la majorité des cas significatives.

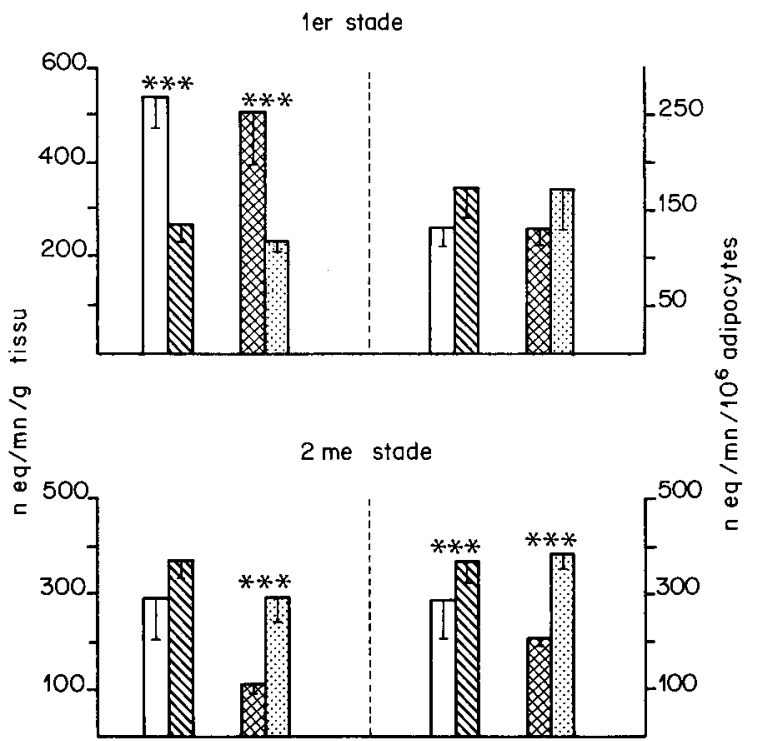

FIG. 4. - Activités lipoprotéine lipase dans le tissu adipeux d'agneaux élevés en plein air ou en bergerie et sacrifiés à $24 \mathrm{~kg}$ (premier stade) ou $32,5 \mathrm{~kg}$ (deuxième stade). Les activités sont rapportées par minute (moyenne \pm erreur-type) soit au gramme de tissu soit à $10^{6}$ adipocytes. Légende et

Comparaison par le test de Mann-Whitney : voir figure 1.

\section{Discussion.}

Nos résultats montrent que chez l'Agneau, élevé en plein air ou en bergerie, la mise en réserve des graisses dans le tissu adipeux est très intense, qu'elle soit le fait de la synthèse de novo des lipides ou de l'action de la lipoprotéine lipase.

L'Agneau en croissance assure l'essentiel de la lipogenèse à partir du tissu adipeux (Ingle, Bauman et Garrigus, 1972 a et b, Vézinhet, 1976, Vernon, 1980). Chez les ruminants, la synthèse de novo des acides gras est en majeure partie liée à l'utilisation de l'acétate, précurseur privilégié de la source de carbone (Hanson et Ballard, 1967, Vézinhet, 1976, Vernon, 1980, Pike et Roberts, 1981). Cette donnée se trouve confirmée dans notre étude, avec cependant une réduction 
très sensible du niveau d'utilisation de l'acétate entre le premier stade d'étude (poids de sacrifice $24 \mathrm{~kg}$ ) et le deuxième (poids de sacrifice $32,5 \mathrm{~kg}$ ). Cette situation est très difficilement explicable. On peut tout d'abord penser. que l'approche du stade adulte se traduit par un net fléchissement de l'activité de synthèse des lipides lié à un état d'engraissement optimum. Par ailleurs, les agneaux abattus à $24 \mathrm{~kg}$ l'étaient à une période de l'année (fin avril, début mai) où les variations de température entre jour et nuit étaient encore très fortes, tandis que les animaux sacrifiés à $32,5 \mathrm{~kg}$ i'étaient à la fin du mois de juin dans des conditions climatiques et d'environnement très différentes. Ceci pourrait justifier une forte variation des comportements métaboliques. La confirmation d'une telle hypothèse nécessiterait une expérimentation en milieu contrôlé, telle qu'une chambre climatisée permettant de préciser le rôle de facteurs comme la température.

On peut aussi penser que les agneaux de plein air, en présence de leur mère, consomment encore une alimentation lactée jusqu'au stade de $24 \mathrm{~kg}$ tandis que cette alimentation serait devenue pratiquement nulle à $32,5 \mathrm{~kg}$. Cette variation dans l'apport nutritionnel peut induire un métabolisme lipidique très différent.

On sait que le glucose peut être utilisé comme précurseur de la synthèse des acides gras chez le ruminant (Ballard, Filsell et Jarret, 1972) bien qu'il soit essentiellement réservé au métabolisme oxydatif (Pike et Roberts, 1981). Dans le cas de l'élevage de plein air il semble que l'utilisation du glucose au premier stade étudié puisse être favorisée pour la biogenèse des acides gras, tandis que l'élevage en bergerie indique un taux d'utilisation du glucose bien plus faible. Au deuxième stade, l'utilisation du glucose est fortement réduite chez les animaux de plein air et reste sensiblement du même ordre pour les agneaux de bergerie. Là encore on peut penser que ces différences s'expliquent par les variations d'environnement et d'apport nutritionnel. Ce dernier facteur concernant la nutrition doit aussi être contrôlé pour permettre d'apporter une réponse qui ne peut être obtenue dans une expérimentation comme celle décrite, laquelle repóse sur l'étude d'activités métaboliques dans des conditions traditionnelles d'élevage avec notamment un accès libre à la nourriture.

Quoi qu'il en soit, on observe avec l'acétate et le glucose que, dans les conditions d'un élevage de plein air, la lipogenèse subit des variations considérables selon le poids auquel est effectuée la mesure. Dans le cas d'un élevage en bergerie, la lipogenèse observée conserve une intensité du même ordre pour les deux stades étudiés. En ce qui concerne les enzymes de la lipogenèse, il ne semble pas y avoir de liaison étroite entre le niveau de synthèse mesuré et les activités enzymatiques. Cela est en particulier vrai pour l'acétyl-CoA-carboxylase dans le cas du tissu omental où on ne retrouve pas lors du premier stade d'étude la nette supériorité de l'activité de synthèse constatée pour l'incorporation de glucose et d'acétate. Pour le tissu sous-cutané les résultats obtenus sont concordants entre les activités enzymatiques et l'activité d'incorporation.

Enfin, lors de l'étude réalisée au deuxième stade, il y a une bonne concordance entre les activités d'incorporation et enzymatiques tant pour l'acétyl-CoA que poúr l'isocitrate déshydrogénase. On note pour l'essentiel que c'est la 
NADPH isocitrate déshydrogénase qui assure chez l'Agneau l'essentiel de la productin de NADPH nécessaire à la biogenèse des acides gras ce qui est conforme aux observations de Bauman et Davis (1975). L'activité de la NADP inalate déshydrogénase, essentielle chez le monogastrique, semble très faible chez le ruminant et tend même à disparaître avec l'âge comme c'est le cas pour les agneaux du deuxième stade.

Enfin, notre étude relative à l'activité de la lipoprotéine lipase révèle que cette dernière contribue à la mise en réserve des graisses dans le tissu adipeux. Cette observation rejoint celle de Haugeback (1973) qui a montré que chez les ovins, sans atteindre les niveaux d'activité constatés chez d'autres espèces, notamment chez les oiseaux, la LPL peut jouer un rôle non négligeable.

Reçu en décembre 1982. Accepté en mars 1983.

\section{Références}

BALLARD J. F., FILSELL O. H., JARRET I. G., 1972. Effects of carbohydrate availability on lipogenesis in sheep. Biochem. J., 102, 952-963.

BAUMAN D. E., DAVIS C. L., 1975. Regulation of lipid metabolism, 496-509. In MAC-DONALD 1. W., WARNER A. C. I., Digestion and metabolism in the ruminant. Univ. New-Engl. Publ. Unit, Armidale, Australia.

BERGMEYER H. U., 1963. Methods of enzymatic analysis. Acad. Press, New York.

BEZMAN A., FELTS J. M., HAVEL R. J., 1962. Relation between incorporation of triglycerides fatty acids and heparin released lipoprotein lipase from adipose tissue slices. J. Lipid Res., 3, 427-431.

CHAKRABARTY K., LEVEILLE G. A., 1969. Acetyl-CoA-carboxylase and fatty acid synthetase activities in liver and adipose tissue of meal-fed-rats. Proc. Soc. exp. Biol. Med., 131, 1051-1054.

CHANG H. C., SEIDMAN I., TEEBOR G., LANE D. M., 1965. Liver acetyl-CoA-carboxylase and fatty acid synthetase : relative activities in the normal stade and in hereditary obesity. Biochem. biophys. Res. Comm., 28, 682-686.

CHILLIARD Y., DORLÉANS M., FEHR R. M., 1977. Mise en évidence d'une activité lipoprotéine lipasique dans le tissu adipeux de chèvre : comparaison de trois méthodes d'extraction. Ann. Biol. anim. Bioch. Biophys., 17, 107-122.

DOLE V. P., MEINERTZ H., 1960. Microdetermination of long chain fatty acids in plasma and tissues. J. biol. Chem., 235, 2595-2599.

FLATT J. P., 1970. Conversion of carbohydrate to fat in adipose tissue : an energy yielding and, therefore, self limiting process. J. Lipid Res., 11, 131-139.

GARFINKEL A. S., BAKER N., SCHOTZ C. M., 1967. Relationship of lipoprotein lipase activity to triglyceride uptake in adipose tissue. J. Lipid Res., 8, 274-280.

HANSON R. W., BALLARD F. J., 1967. The relative significance of acetate and glucose as precursors for lipid synthesis in liver and adipose tissue from ruminants. Biochem. J., 105, 529-536.

HAUGEBACK C., 1973. Effects of nutrition and age on adipose tissue growth and development in lambs. Th. Univ. Missouri, pp. 169.

HSU R. Y., LARDY H. A., 1969. Malic enzyme, 220-235. in LOWENSTEIN J. M., Methods in enzymology. Vol. XVII, Acad. Press, New York, London.

INGLE D. L., BAUMAN D. E., GARRIGUS U. S., 1972a. Lipogenesis in the ruminant : in vitro study of tissue sites, carbon source and reducing equivalent generation for fatty acid synthesis. $J$. Nutr., 102, 609-616. 
INGLE D. L., BAUMAN D. E., GARRIGUS U. S., 1972b. Lipogenesis in the ruminant : in vivo site of fatty acid synthesis in sheep. J. Nutr., 102, 617-624.

MARTIN D. B., VAGELOS P. R., 1965. Fatty acid synthesis in adipose tissue, 211-216. In A. E. RENOLD, G. F. CAHILL, Handbook of physiology, Section 5.211. Am. Physiol. Soc., Washington D.C.

NOUGUESS J., VÉZINHET A., 1977. Evolution pendant la croissance de la cellularité du tissu adipeux chez le lapin et l'agneau. Ann. Biol. anim. Bioch. Biophys., 17, 799-806.

PIKE B. $\forall .$, ROBERTS C. J., 1981. Comparison of glucose and acetate as substrates for lipid synthesis in bovine adipocytes. Res. vet. Sci., 80, 390-391.

POTHOVEN M. A., BEITZ D. C., 1973. Effect of adipose tissue site, animal weight, and longterm fasting on lipogenesis in the bovine. J. Nutr., 103, 468-475.

ROBINSON D. S., 1963. Clearing factor lipase and its action in the transport of fatty acids between the blood and the tissues. Adv. Lipid Res., 1, 133-181.

RODBELL M., 1964. Metabolism of isolated fat cells. I. Effects of hormone on glucose metabolism and lipolysis. J. biol. Chem., 239, 375-380.

SCOW R. O., HAMOSH M., BLANCHETTE-MACKIE E. J., EVANS A. J., 1972. Uptake of blood triglyceride by various tissues. Lipids, 7, 497-505.

VERNON R. G., 1980. Comparative aspects of lipid metabolism in monogastric, pre-ruminant and ruminating animals. Bioch. Soc. Trans., 8, 291-293.

VÉZINHET A., 1976. Etude du tissu adipeux chez l'agneau et le lapin après la naissance: développement, lipolyse, lipogenese, influence de l'hypophysectomie et de l'hormone de croissance. Th. Dr. es. Sci., Montpellier, 167 pp.

YOUNG J. W., THORP S. L., de LUMEN H. Z., 1969. Activity of selected gluconeogenic and lipogenic enzymes in bovine rumen mucosa, liver and adipose tissue. Biochem. J., 114, 83-88. 